\title{
Revisión sistemática del desempeño docente en la educación
}

Systematic review of teacher performance in education.

\section{Mg. Lourdes Ana Cotaquispe Guzmán}

Doctoranda en Educación por la Universidad César Vallejo. Maestra en Educación con mención en Docencia y Gestión Educativa por la Universidad César Vallejo, Lima, Perú. Licenciada en Educación por la Universidad Nacional Mayor de San Marcos. Subdirectora de Formación General en la I.E.E. José Granda.Cel. 963716177, Email: lcotaquispe@ucvvirtual.edu.pe ORCID: https://orcid.org/0000-0003-3087-5409, Google académico: https://scholar.google.es/citations?user=0vuGCK4AAAAJ\&amp;hl=es

\section{Dr. Juan Pedro Soplapuco Montalvo}

Doctor en Ciencias de la Educación, Maestro en Docencia Universitaria e Investigación Educativa, Licenciado en Educación, por la Universidad Nacional Pedro Ruiz Gallo de Lambayeque, Perú. Docente de la Escuela de Posgrado de la Universidad Cesar Vallejo, Chiclayo. Email: smontalvojp@ucvvirtual.edu.pe, Cel.949570072, ORCID: https://orcid.org/0000-0003-4631-8877, Google académico: https://scholar.google.com/citations?user=Z6cSZVgAAAAJ\&hl=es

\section{Mg. Ana Beatriz Rivas Moreano}

3Maestra en administración de la educación por la Universidad Cesar Vallejo, sede Lima este, Profesora de Educación primaria en el instituto superior pedagógico público de Puquio, Ayacucho, Perú, Docente de pregrado en la Universidad Autónoma del Perú, Lima. Docente en institución educativa Pública Email arivasmor@ autonoma.edu.pe, Cel. 949702751, ORCID 0000-0003-2633-5717, Google académico https://scholar.google.es/citations?view_op=new_profile\&hl=es

\section{Mg. Julia Rosario Vales Gonzales}

4Maestra en Gestión Educativa y Docencia Universitaria, Universidad César Vallejo, Lima, Perú. rosariovalesg@gmail.com, ORCID: 0000-0002-9364-6163, https://scholar.google.es/citations?user=EnrV0NQAAAAJ\&hl=es

\section{Revista Iberoamericana de la Educación \\ Vol-Especial 12021 \\ e-ISSN: 2737-632x}

RESUMEN: El desempeño docente es el conjunto de acciones realizadas por el docente, que involucra diversos aspectos, establece relaciones entre el docente, estudiante y su entorno. El trabajo del docente es un factor que conlleva a la calidad educativa. Objetivo. Identificar lo que significa el desempeño docente, de acuerdo a la información encontrada en las bases de datos como Scielo, Redalyc, Latindex, Scopus, EBSCO y Dialnet, en el 
período del 2017 al 2021, en los niveles, primaria, secundaria y superior. Materiales y métodos. Se realiza un estudio cuantitativo y de manera descriptiva, con la revisión de 28 artículos, de los últimos cuatro años. Resultados. Aplicados los criterios de inclusión y exclusión se seleccionaron 28 artículos potenciales, en los cuales se revisaron las investigaciones realizadas sobre desempeño docente. Conclusión. El desempeño docente comprende las acciones desarrolladas por el docente, que involucra múltiples aspectos y de significado variado para los investigadores, siendo materia de continuas investigaciones. Palabras clave: Desempeño docente, educación, calidad educativa, aprendizaje.

ABSTRACT: The teaching performance is the set of actions carried out by the teacher, which involves various aspects, establishes relationships between the teacher, student and their environment. The teacher's work is a factor that leads to educational quality. Objective. Identify what teaching performance means, according to the information found in databases such as Scielo, Redalyc, Latindex, Scopus, EBSCO and Dialnet, in the period from 2017 to 2021, at the primary, secondary and higher levels. Materials and methods. A quantitative and descriptive study is carried out, with the review of 28 articles, from the last four years. Results. Applying the inclusion and exclusion criteria, 28 potential articles were selected, in which the research carried out on teaching performance was reviewed. Conclution. The teaching performance includes the actions developed by the teacher and that involves multiple aspects, and of varied significance for researchers, being the subject of continuous research. key words: Teaching performance, education, educational quality and learning. 


\section{INTRODUCCIÓN}

Los cambios constantes que se presentan en el mundo, hacen surgir la necesidad de reflexionar sobre la educación que necesitan los estudiantes de hoy, si el rol que desempeñan los educadores son los adecuados, en el contexto en la que actualmente se vive es una necesidad aplicar estrategias y recursos acordes al trabajo realizado, tal como lo sustenta Mendoza, (2020), ¿cómo debe ser el desempeño de los docentes?, analizar lo que debe cumplir el docente en su quehacer en la escuela.

En los últimos años surge una preocupación con respecto al desempeño de la profesión docente, organismos tales como UNESCO, OEA, Banco Mundial han llevado a cabo diversos estudios e investigaciones sobre la formación del docente, para mejorar su desempeño, inclusive implementar sistemas de evaluación del docente UNESCO (2013). Asimismo, UNESCO (2007), considera que el trabajo del docente es un factor especial en la calidad educativa, la cual se relaciona con el buen uso de los recursos, la equidad en el servicio y responder a las necesidades de la sociedad y de los estudiantes, garantizando el acceso a la educación y que el estudiante pueda concluir satisfactoriamente el e nivel educativo

En la misma línea el informe del BID (2020) reconoce la calidad docente como un elemento clave para mejorar la calidad educativa en América Latina., dicho informe presenta cinco claves importantes a ser tomados en cuenta, tales como elaborar estándares de desempeño docente, plantear los objetivos a lograr, elaborar instrumentos para la evaluación los cuales pueden ser más de uno y los resultados deben permitir la toma de decisiones para la mejora de la calidad educativa.

Los estudiosos del tema señalan que el desempeño docente se relaciona con la calidad de la educación Escribano (2018), Soria et al. (2021), Montenegro (2007), mencionan que el trabajo docente es un elemento que se relaciona con 
el aprendizaje de los estudiantes, inclusive remarca que es independientemente de la situación económica de la que dispone el docente, aunque según UNESCO (2007) sostiene que se revalore el trabajo del docente, considerando una remuneración justa y aplicación de políticas que permitan el desarrollo profesional. Por lo descrito surge la necesidad de definir las características que debe reunir el docente, estableciendo un perfil que comprende competencias y capacidades.

Nuestro país no es ajeno ante la situación descrita a finales del año 2000, se consideró la problemática docente en el centro de la agenda educativa nacional. En el año 2012, el Concejo Nacional de Educación entrega al ministerio de educación, el documento del buen desempeño docente para su revisión con participación de instituciones de gestión descentralizada, centros de formación y especialistas, del cual surge el Marco del buen desempeño docente que viene a constituir el instrumento que orienta la política educativa peruana, que según Gálvez y Milla (2018) es un avance para mejorar la práctica pedagógica. Minedu (2012), el MBDD consta de cuatro dominios, nueve competencias y cuarenta desempeños, los cuales definen al maestro que necesita el país.

Asimismo, Minedu (2019) en su plataforma de evaluación docente informa que, en dicho año, en la evaluación para ingreso a la carrera pública magisterial, de 230,952 docentes inscritos, solamente 15,874 aprobaron la prueba y clasificaron para la siguiente fase, ello indica que también se tiene que mejorar en el dominio de los conocimientos propios de la profesión. Diversos factores se asocian a los bajos logros de aprendizaje, siendo uno de ellos el desempeño docente pues tal como sostiene (Escribano, 2018), por cuanto el desempeño docente es un punto clave en la calidad de la educación, entonces es necesario tomar en cuenta el desempeño de los maestros. 
Por lo anteriormente descrito, el presente estudio aborda la investigación del significado del término desempeño docente, por cuanto es necesario conocer como actualmente se concibe lo que es el desempeño docente, características de dicho desempeño, que aspectos o que factores deben ser tomados en cuenta en dicha labor,

\subsection{DESEMPEÑO DOCENTE}

Para entender el término desempeño docente se ha hecho uso de la literatura sobre el tema, se toma en consideración a Montenegro (2007) quien define al desempeño docente como un conjunto de acciones sistematizadas que involucra planificación, desarrollo de evaluación y seguimiento de procesos curriculares. En el rol de docente se hace necesario tener competencias específicas en cuatro campos, los cuales son: ámbito institucional, pedagógica e intrapersonal, ello le permite cumplir su labor de manera eficiente. Para Cuenca (2011) es una función de relación mutua porque en su atención al estudiante se establece relaciones con otras personas tales como los demás profesores, padres de familia y la sociedad, su vez Casas (2019) hace alusión al cumplimiento de funciones, también menciona que se establece relaciones entre docente, estudiante y su medio.

El término desempeño tiene que ver con la actuación de la persona, se mide los factores que afectan y el logro alcanzado según Rodríguez y Lechuga (2019), implica tener metas y resultados. Asimismo, diversos estudiosos tales como Gálvez y Milla (2018), Álvarez (2020), Martínez et al. (2021), Campos et al. (2021) coinciden en señalar que es durante la práctica docente cuando se muestra la competencia en su labor y su efecto que tiene en el logro de los aprendizajes.

Murillo (2012), Campos et al. (2021), Geraldo et al. (2021) hace referencia a la importancia de la motivación como uno de los elementos necesarios que 
impulsa a lograr una educación de calidad, buen desempeño del docente, cambiar las prácticas tradicionales, este último establece tres tipos de motivación: intrínseca referida a las necesidades y emociones, extrínseca que se relaciona con la responsabilidad y el ser competente, por último la motivación trascendente que es cuando se logra la satisfacción personal al ayudar a otras personas. Peniche (2020) menciona que otro aspecto a tomar en cuenta es el compromiso del docente, lo cual se relaciona con el mejoramiento del desempeño del profesor. Puertas (2018) menciona la importancia de la regulación de las emociones. Los factores afectivos inciden en el desempeño docente según Gallardo y Pacheco (2019) los cuales se manifiestan en la motivación por su trabajo. More y Morey (2021) sustentan la importancia del clima institucional para el buen desempeño docente, lo que también abarca a la motivación y logros de aprendizaje en los estudiantes. Por otro lado, Trujillo (2020) menciona que no existe una correlación significativa entre la satisfacción laboral y el desempeño, porque entra en juego otros factores como exceso de tareas, escaso interés de los estudiantes. Cuando mencionan el desempeño docente, destacan la actividad de planificación que realiza el docente, aplicando estrategias pedagógicas, la cual debe ser motivo de preocupación en la gestión pedagógica Quispe (2020), ello concuerda con Gálvez y Milla (2018), Urrutia (2020), Martínez y Gómez. (2021), Hernández y Ceniceros (2018), Álvarez (2020), quienes señalan que el docente debe tener manejo de la preparación de los aprendizajes, siendo uno este uno de los dominios de Marco del Buen Desempeño Docente, para lo cual se requiere tener en cuenta las características de los estudiantes y la planificación, manifiesta Valcazar (2020) sin dejar de lado la diversidad y la inclusión, en esta etapa se realiza la aplicación de teorías pedagógicas estrategias y recursos para ser aplicados en el aula. García (2020) añade que cuando se cumple lo planificado en el 
diseño curricular repercute en un mejor desempeño docente, por ello también tomar en cuenta Achata y Quispe (2018) cuando afirma que el profesor que conoce el currículo nacional evidencia mejor desempeño, Haddad y Barboza (2020) agrega que en el trabajo remoto requiere más tiempo para la planificación.

Cuando el docente planifica la actividad a desarrollar con los estudiantes, toma en cuenta las estrategias de enseñanza, según los autores es uno de los factores que influyen en el desempeño docente según lo sustentado por Hernández y Ceniceros. (2018), Quispe (2020), que en el proceso de aprendizaje se desarrolla actividades de tipo experimental, productivas y comunicativas, agrega Soria et al. (2020) que la adecuada aplicación de estrategias en la enseñanza no es el único factor que permite tener altos logros de aprendizaje.

El desempeño docente está relacionado con la actuación del docente como profesional, que pone en juego sus capacidades para tener un control de sus acciones, las capacidades personales que tiene el docente le permiten tener un control de sus acciones según refiere Hernández y Ceniceros (2018). Osorio (2020) agrega que el docente debe ser un ejemplo a seguir, concuerda en ello Martínez et al. (2021), Trujillo et al. (2020), Campos et al. (2021), quienes señalan que es en la práctica pedagógica, en el desarrollo de la enseñanza, donde se observa realmente el desempeño del profesor, durante dicha etapa de su labor, muestra los recursos psicosociales destrezas y las actitudes lo que conforma su conducta en la enseñanza, considerando además las habilidades comunicativas en su relación con los estudiantes. Chinet et al. (2017) se refiere a la identidad cultural del docente como un factor que permite tener un mejor desempeño en su labor.

Diversos autores señalan la relación del uso de la tecnología y el desempeño docente, Mendoza (2020), Gonzales (2019) señalan que se requiere hacer uso 
de la tecnología de acuerdo a las necesidades, no es posible enseñar a las nuevas generaciones con los mismos recursos de la enseñanza tradicional, en la educación virtual también cumple un rol importante el docente en el desarrollo de la planificación y ejecución de la experiencia de aprendizaje, utilizando de manera adecuada la comunicación y la información Picón et al. (2020). El maestro necesita preparación y entrenamiento en el uso de herramientas digitales, Granados (2019), Galán (2020) que las tecnologías por sí solas no cumplen a cabalidad el proceso de enseñanza porque se necesita la aplicación de estrategias de enseñanza y aprendizaje, mediante actividades que involucran participación de los estudiantes. Al respecto Fuentes et al. (2019) agrega que los docentes no cuentan con suficientes destrezas referidas a la competencia digital. Huamán et al (2021) dice que en la actualidad con el trabajo remoto que desarrollan los docentes se ha demostrado que se necesita fortalecer las competencias digitales de los maestros.

Casas (2019) menciona la relación que existe entre el liderazgo del director y el desempeño docente, precisa que la gestión pedagógica debe estar enfocada en mejorar los aprendizajes, brindando recursos económicos, tecnológicos, que responda a las necesidades y cambios, al respecto según el marco del buen desempeño directivo también hace referencia que el directivo debe tener liderazgo en el campo pedagógico que permita orientar, conducir y consolidar la gestión escolar. López et al. (2019) destaca la gestión directiva especialmente en la construcción de espacios formativos, que promueva el trabajo en equipo y de manera colaborativa para lograr los objetivos y metas. También, De la Cruz (2018) manifiesta que el liderazgo es importante en el quehacer de las personas y con mayor énfasis en el desempeño docente, inspirando a los estudiantes a desarrollar el liderazgo. 
En este contexto, se entiende que el liderazgo es un factor importante en relación a las diversas actividades profesionales del ser humano, más aún en el desempeño docente; el liderazgo transformacional cambia situaciones y personas, inspira a los estudiantes a ser líderes de este proceso. Esta es la razón fundamental para describir y correlacionar el liderazgo transformacional y el desempeño docente en el Cepre-UNCP. Y la presente investigación contribuye a establecer que el liderazgo transformacional influye significativamente en el desempeño docente en este contexto, se entiende que el liderazgo es un factor importante en relación a las diversas actividades profesionales del ser humano, más aún en el desempeño docente; el liderazgo transformacional cambia situaciones y personas, inspira a los estudiantes a ser líderes de este proceso. Esta es la razón fundamental para describir y correlacionar el liderazgo transformacional y el desempeño docente en el Cepre- UNCP. Y la presente investigación contribuye a establecer que el liderazgo transformacional influye significativamente en el desempeño docente En este contexto, se entiende que el liderazgo es un factor importante en relación a las diversas actividades profesionales del ser humano, más aún en el desempeño docente; el liderazgo transformacional cambia situaciones y personas, inspira a los estudiantes a ser líderes de este proceso. Esta es la razón fundamental para describir y correlacionar el liderazgo transformacional y el desempeño docente en el Cepre-UNCP. Y la presente investigación contribuye a establecer que el liderazgo transformacional influye significativamente en el desempeño docente.

Asimismo diversos autores coinciden en señalar la importancia de la formación continua en la labor del docente, Álvarez (2020), Peniche et al. (2020), Mendoza (2020), García (2020), López et al. (2019), diferenciando en la participación en los cursos por cuenta propia y en los ofrecidos por la 
escuela y otras instituciones, también se considera el aprendizaje colectivo, intercambio de ideas y experiencias, con el apoyo de personal calificado también complementa López et al (2019) sobre realizar capacitaciones para los docentes especialmente en lo referente a recursos didácticos. Gómez y Valdez (2019) sostiene que un modo para lograr el desarrollo continuo del docente es mediante la evaluación.

También, otro aspecto a mencionar es la relación del desempeño docente con la gestión directiva tal como lo manifiesta López, et al. (2019) que es necesario construir espacios que permitan el desarrollo de buenas prácticas de los maestros y estudiantes, logrando el cumplimiento de metas. A su vez Meza et al. (2021) hace mención a la gestión directiva en sus diversas dimensiones tales como pedagógica, institucional, administrativa y comunidad incide en el desempeño docente, además More y Morey (2021) resalta el rol del directivo en la gestión del clima institucional que tiene relación positiva con el desempeño del docente.

Diversos autores señalan que la práctica pedagógica es mejorada por el acompañamiento pedagógico la cual se entiende como las acciones de asesoría que realizan los especialistas y el equipo directivo de la instituciones educativas, para mejorar las practicas llevadas a cabo por los docentes, para ello el punto de partida es la observación de la clase, promoviendo el dialogo reflexivo para detectar fortalezas, debilidades y en base de ello llevar a cabo las orientaciones y asesorías respectivas para fortaleces las capacidades y competencias del docente según lo expuesto por MINEDU (2014), Gálvez y Milla (2018), por otro lado Agreda y Pérez (2020) sustenta que durante el acompañamiento se debe promover la reflexión crítica del mismo docente, lo que le permite mejorar su práctica pedagógica. 


\section{MATERIALES Y MÉTODOS}

El presente estudio de revisión sistemática de la bibliografía del tema desempeño docente, se aplicó el método de revisión documental, que según Gómez (2017) es una herramienta que permite construir el conocimiento, ampliar los constructos y enriquecer el vocabulario. Se organizó los datos en tablas y figuras, además de comparaciones de los hallazgos encontrados.

Con respecto a los criterios de inclusión considerados en el estudio sobre el desempeño docente fueron materia de revisión las bases de datos de Scopus, SciELO, EBSCO, Latindex, Dialnet y Redalyc en los cuales se consideró las publicaciones entre los años 2017 hasta el mes de mayo del 2021, artículos científicos de tipo cuantitativo y cualitativo, realizados en diversos niveles educativos, los resultados fueron ordenados en una matriz de Excel, tomando en cuenta el título, revista, tipo de investigación, año de publicación, muestra, resultados y conclusiones.

Los criterios de exclusión tomados en consideración fueron aquellos, resumen de congresos, cartas al editor, ponencias e informes.

En cuanto a los descriptores utilizados son educación, calidad educativa y desempeño docente, las revistas revisadas son de diversos países tales como México, España, Paraguay, Ecuador, Brasil, y Perú, con respecto a los idiomas de las publicaciones corresponden al español y portugués.

\section{RESULTADOS}

En la revisión bibliográfica se consideró artículos científicos, ensayos, textos escritos por teóricos y especialistas del tema. De los 50 artículos de revisión sistemática ... se aplicó los criterios de exclusión e inclusión, considerando finalmente 28 , en cuanto a los idiomas en que fueron publicados, 27 son de idioma español y 1 en idioma portugués. 
En la figura 1, se observa, las publicaciones de los artículos por países, como resultado se tiene que Perú es el país que presenta mayor número de publicaciones con respecto al desempeño docente, seguido de México y España, con menor número de publicaciones se tiene a Paraguay, Brasil y Ecuador.

Figura 1

Publicación de artículos por países

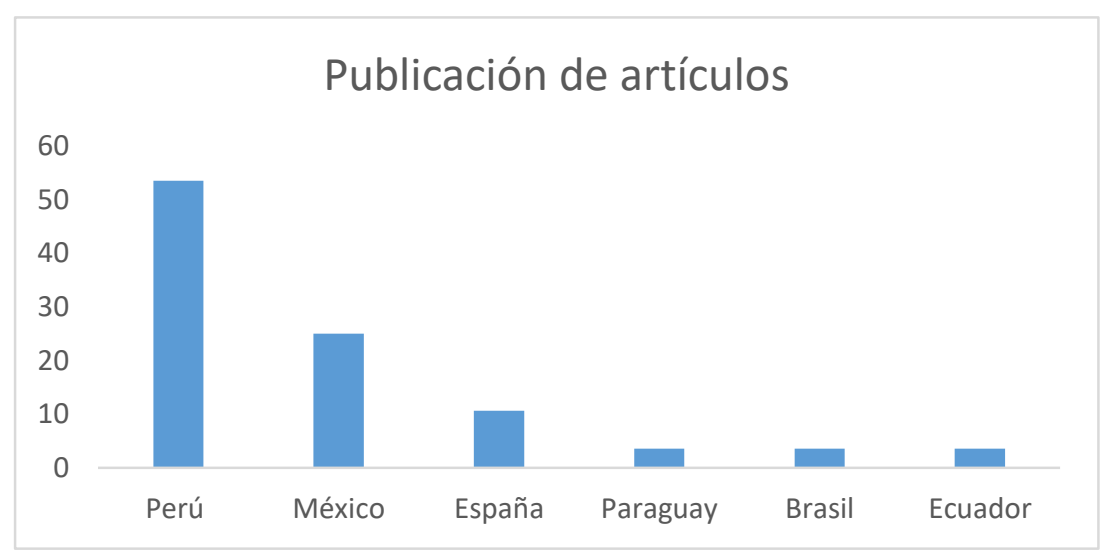

En la tabla 1 se muestra la distribución de las publicaciones por países, siendo Perú el país que presenta mayor número de publicaciones, seguido de México, siendo los países que mas destacan a nivel de américa.

Tabla 1

Distribución de publicaciones de artículos por países.

\begin{tabular}{lcc}
\hline Países & No Publicaciones $^{\text {Porcentaje }}$ \\
\hline Perú & 15 & 53.5 \\
México & 7 & 25.0
\end{tabular}




\begin{tabular}{lcc} 
España & 3 & 10.7 \\
Paraguay & 1 & 3.6 \\
Brasil & 1 & 3.6 \\
Ecuador & 1 & 3.6 \\
\hline Total & 28 & $100 \%$
\end{tabular}

En la figura 2 se observa la publicación de artículos por Base de datos, destacando Scielo con mayor porcentaje de artículos publicados y Dialnet con menor porcentaje de artículos publicados

Figura 2

Artículos publicados por base de datos

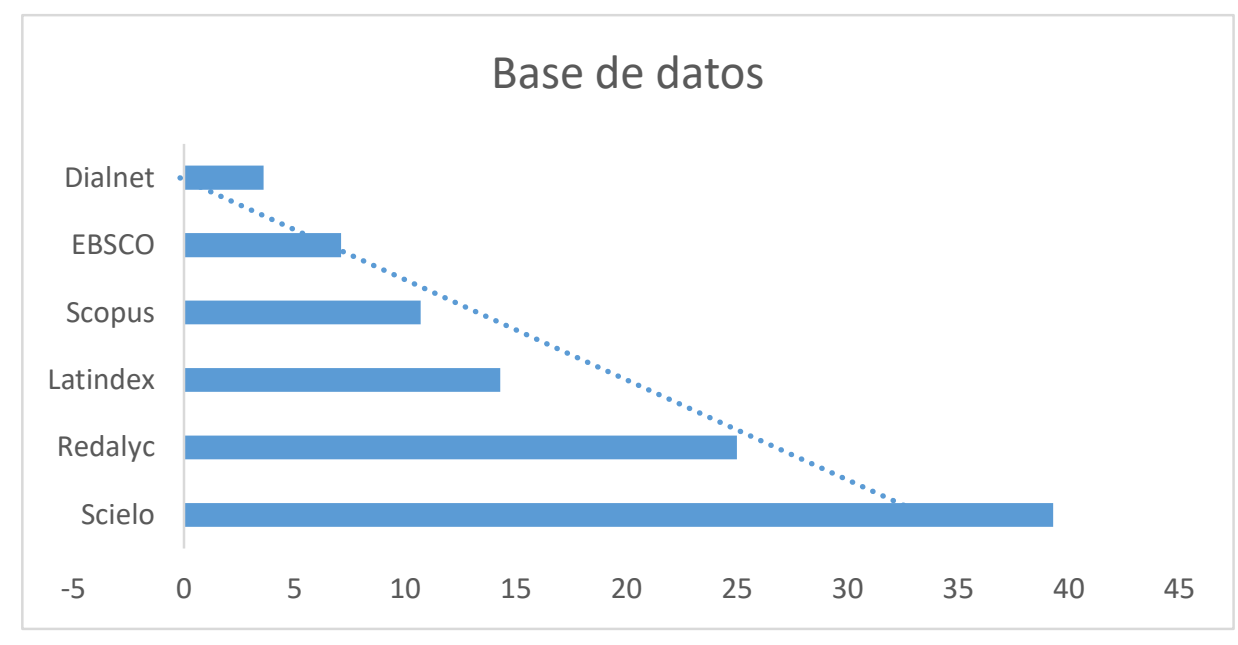

En la tabla 2, se muestra las bases de datos de los artículos publicados, siendo Scielo la que cuenta con mayor número de artículos publicados, en los últimos cinco años, seguido de Redalyc, con menor cantidad de publicaciones se tiene EBSCO y Dialnet. 
Tabla 2

Publicaciones de artículos por bases de datos

\begin{tabular}{lcccc}
\hline \multicolumn{1}{c}{ Base de Datos } & \multicolumn{2}{c}{ Frecuencia } & \multicolumn{2}{c}{ Porcentaje } \\
\hline Scielo & 11 & \multicolumn{2}{c}{39.3} & \\
Redalyc & & 7 & \multicolumn{2}{c}{25.0} \\
Latindex & 4 & & 14.3 & \\
Scopus & 3 & & 3.7 & \\
EBSCO & & 2 & 3.6 & \\
Dialnet & 1 & & & \\
\hline
\end{tabular}

$-$

Total

28

$100 \%$

En la figura 3 se observa los niveles educativos en los cuales se realizó las investigaciones, se evidencia que el mayor porcentaje de artículos publicados corresponde al nivel educativo secundaria, seguido del nivel superior y en el nivel primaria se ha realizado menor porcentaje de investigaciones.

Figura 3

Artículos por nivel educativo 


\section{Nivel Educativo}

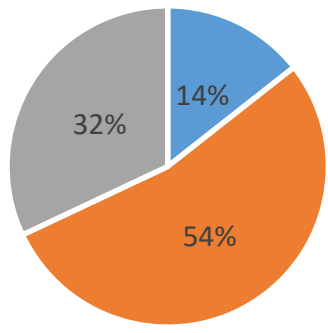

- Primaria $₫$ Secundaria $₫$ Superior

En la tabla 4 se muestra los autores, año de publicación y los hallazgos

encontrados en las investigaciones realizadas.

Autores y Hallazgos en cada investigación

\begin{tabular}{|c|c|c|c|}
\hline \multicolumn{2}{|c|}{$\mathbf{N}^{0}$. Autor } & \multirow{2}{*}{ 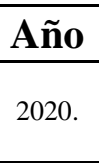 } & \multirow{2}{*}{$\begin{array}{l}\text { Hallazgos } \\
\text { Entre los aspectos que tiene efecto en el desempeño docente, se encuentra las } \\
\text { cuestiones propias que se relaciona con el compromiso del profesor y además el } \\
\text { clima organizacional y los recursos pedagógicos. }\end{array}$} \\
\hline 1 & Peniche et al. & & \\
\hline 2 & Urrutia. & 2020 & $\begin{array}{l}\text { Para un buen desempeño docente es necesario fortalecer la preparación de los } \\
\text { docentes en cuanto a aspectos tales como: pedagógicos, didáctica y en la parte } \\
\text { organizativa. }\end{array}$ \\
\hline 3 & Geraldo et al. & 2021 & $\begin{array}{l}\text { La motivación repercute en la práctica que realiza el docente, es importante el ánimo } \\
\text { que tiene el docente, permite que su labor resulte fructífera, cuando tiene metas altas } \\
\text { su desempeño mejora. }\end{array}$ \\
\hline 4 & Mendoza & 2020 & $\begin{array}{l}\text { Un buen desempeño docente implica el aprendizaje continuo porque el contexto } \\
\text { cambia y surge la necesidad de la adquisición de competencias para el conocimiento } \\
\text { y uso de la tecnología, sumamente necesarias de acuerdo a las necesidades, }\end{array}$ \\
\hline 5 & Meza et al. & 2021 & $\begin{array}{l}\text { La elaboración y ejecución de los proyectos en la escuela y el fortalecimiento de los } \\
\text { procesos pedagógicos son aspectos considerativos en el desempeño del profesor. La } \\
\text { gestión en la escuela, toma en cuenta varios aspectos tales como: institucional, } \\
\text { pedagógica, administrativa y comunitaria }\end{array}$ \\
\hline 6 & Álvarez & 2020 & $\begin{array}{l}\text { Cuando se desea medir el desempeño docente, se toma en consideración el } \\
\text { conocimiento y manejo de los procesos pedagógicos, como acciones que desarrolla el } \\
\text { docente para el logro de las competencias, tales como recojo de saberes previos, } \\
\text { motivación, conflicto cognitivo, procesamiento de la información }\end{array}$ \\
\hline 7 & Martínez et al. & 2021 & $\begin{array}{l}\text { El desempeño docente se observa en la práctica profesional, cuando se realiza el } \\
\text { manejo de situaciones que se producen en el aula, trabajando un plan de acción para } \\
\text { transformar las dificultades en fortalezas. }\end{array}$ \\
\hline
\end{tabular}




\begin{tabular}{lll}
\hline No. Autor & Año & \multicolumn{1}{c}{ Hallazgos } \\
\hline 8 Hernández y Cenicero & 2018 & $\begin{array}{l}\text { El desempeño docente requiere la práctica de la autoeficacia que favorece la } \\
\text { motivación, siendo positivo en lo que hace, las capacidades que tiene la persona le } \\
\text { permiten tener un control de las acciones tales como pensamientos, comportamientos } \\
\text { que pone en juego al realiza su labor, lo que se traduce en un rendimiento efectivo. }\end{array}$ \\
\hline 9 Gálvez y Milla & $2018 \quad \begin{array}{l}\text { En la evaluación del desempeño docente, es necesario prestar atención en lo referente } \\
\text { a la preparación para el aprendizaje de los estudiantes, para lo cual realiza la } \\
\text { planificación de lo que realiza en clase, llámese programación anual, unidades de } \\
\text { clase y las sesiones correspondientes, sin dejar de lado el monitoreo y } \\
\text { acompañamiento al profesor, para lo cual se promueve la reflexión que realiza el } \\
\text { docente sobre su práctica pedagógica, identificando dificultades que serán motivo de } \\
\text { apoyo para su mejora. }\end{array}$ \\
\hline
\end{tabular}

10 Trujillo et al.

2020

11 Soria et al

niveles
cuenta.

12 Gallardo et al

2019

El docente al realizar su labor pone en práctica la afectividad que son los

Se determinó que no existe una correlación significativa entre la satisfacción laboral y del desempeño del profesor pues surgen otras situaciones tales como a pesar de tener motivación por la parte económica sin embargo el exceso de tareas, escaso interés de los estudiantes, repercuten en el desempeño del profesor.

En el buen desempeño docente entra en juego la preparación constante del profesor, el uso de adecuadas estrategias en el aula, además del contexto, ello incide en los niveles del desempeño del profesor, pero no es el único factor a ser tomado en cuenta. factor propicia un ambiente favorable para los aprendizajes, lo que se traduce en mejores resultados en su quehacer.

La puesta en práctica del currículo, la preparación de lo que se desarrolla en la clase,

13 García

2020 la metodología aplicada, el manejo de la evaluación, son características que muestran el trabajo que realiza el profesor, el cual es positivo de acuerdo a como ejecuta lo mencionado.

14 Quispe

2020

2019

16 Chenet et al

2017

17 Picón et al

2020
La relación entre el desempeño docente y la gestión realizada por el directivo, es motivo de investigación, el director siendo el líder pedagógico toma decisiones para la mejora de las prácticas que realiza el docente, promueve la planificación y ejecución de proyectos que coadyuvan a la mejora de los resultados de los aprendizajes.

El trabajo que realiza el directivo, es en dos campos: el administrativo y el pedagógico, éste último es el que repercute principalmente en los aprendizajes, con la puesta en práctica del monitoreo y acompañamiento permite detectar dificultades y desarrollar su mejora, brindando las condiciones adecuadas para el buen desempeño de los docentes

El desempeño docente implica la necesidad de la identidad que practica el docente en su ejercicio laboral tener el conocimiento básico de la materia y saber articular con el contexto produce mejores resultados en el desempeño mostrado por los profesores.

El desempeño docente en el modo del trabajo remoto, muestra la necesidad de recurrir al uso de la tecnología como medio fundamental para la interacción con los estudiantes, esta interacción es llevada a cabo de manera sincrónica y asincrónica, pero todavía los docentes necesitan aprender mucho de la aplicación de la tecnología, lo cual es una tarea pendiente de parte de las autoridades. 


\begin{tabular}{lll}
\hline No. Autor $^{0}$ & Año & Hallazgos \\
\hline
\end{tabular}

18 Galán et al.

2020

En el proceso de aprendizaje no es suficiente que el estudiante tenga acceso a la tecnología, el uso de este recurso necesita de la actuación del maestro, pues se complementa con las adecuadas estrategias que aplica el docente para el logro de los aprendizajes.

\begin{tabular}{|c|c|c|c|}
\hline 19 & De la Cruz & 2018 & $\begin{array}{l}\text { El buen desempeño del maestro, requiere de una visión clara, capaz de generar } \\
\text { cambios, si el docente tiene bajo nivel de motivación genera deficiente desempeño } \\
\text { del docente-. }\end{array}$ \\
\hline 20 & Achata y Quispe & 2018 & $\begin{array}{l}\text { El currículo nacional es un documento base para realizar la planificación es } \\
\text { indispensable que el docente maneje los conocimientos sobre dicho documento con } \\
\text { ello se logra un mejor desempeño del maestro en el aula. }\end{array}$ \\
\hline 21 & Granados et al. & 2019 & $\begin{array}{l}\text { La formación del docente como de cualquier otro profesional, no culmina con los } \\
\text { estudios de pre grado, es indispensable para estar de acorde a los cambios que se } \\
\text { produce en el contexto para capacitarse, actualizarse en los conocimientos, } \\
\text { especialmente de la tecnología. }\end{array}$ \\
\hline 22 & Haddad y Barbosa & 2020 & $\begin{array}{l}\text { En el trabajo remoto que realiza los profesores, se necesita más tiempo para realizar } \\
\text { actividades de planificación, aunque ello implica llevar a cabo un proceso de } \\
\text { reorganización de las prácticas tradicionales. }\end{array}$ \\
\hline 23 & Fuentes et al. & 2019 & $\begin{array}{l}\text { Los docentes que atienden al nivel primaria, muestran tener mejor manejo de las } \\
\text { tecnologías digitales, los cuales son aplicados en sus sesiones, la mayoría de docentes } \\
\text { tiene desde ya un compromiso por aprender y aplicar la tecnología digital con los } \\
\text { estudiantes. }\end{array}$ \\
\hline
\end{tabular}
estudiantes.

24 More y Morey $2021 \quad \begin{aligned} & \text { El desempeño docente se relaciona de manera significativa con la gestión del } \\ & \text { institucional, en lo referente al liderazgo que ejerce el director, motivación, } \\ & \text { reciprocidad y participación en las diferentes actividades tanto de directivos, } \\ & \text { profesores y estudiantes. }\end{aligned}$

\begin{tabular}{|c|c|c|c|}
\hline 25 & Valcázar & 2020 & $\begin{array}{l}\text { En el desempeño que realiza el docente en el aspecto de la planificación de la clase, } \\
\text { se considera la práctica de inclusión, para ello dosifica el contendido, realiza la } \\
\text { retroalimentación de acuerdo a las características y necesidades de los estudiantes, } \\
\text { dando respuesta a la diversidad que se presenta en el aula. }\end{array}$ \\
\hline 26 & Gómez y Valdés & 2019 & $\begin{array}{l}\text { El desempeño docente se mide a través de la evaluación, como una necesidad para } \\
\text { promover el desarrollo profesional permanente del docente, aplicando la reflexión de } \\
\text { como realiza su labor, de tal manera que le permita avanzar en su mejora, tres } \\
\text { procesos a ser tomados en cuenta, la evaluación por si mismo, la evaluación entre } \\
\text { pares y la heteroevaluación. }\end{array}$ \\
\hline 27 & López et al. & 2019 & $\begin{array}{l}\text { El director en el cumplimiento de sus funciones toma en cuenta los resultados de los } \\
\text { aprendizajes, la convivencia y el clima organizacional, elaborando un perfil que va de } \\
\text { acorde a los desafíos del contexto, todo ello va a potenciar la práctica educativa que } \\
\text { realizan los docentes y mejorar los aprendizajes. }\end{array}$ \\
\hline 28 & Agreda y Pérez & 2020 & $\begin{array}{l}\text { El desempeño docente se relaciona con el monitoreo y acompañamiento, este último } \\
\text { permite registrar las evidencias del desempeño, las cuales son analizadas para } \\
\text { elaborar las preguntas que conllevan al diálogo reflexivo realizado por el mismo } \\
\text { docente, que propone nuevos saberes y la transformación de su práctica pedagógica. }\end{array}$ \\
\hline
\end{tabular}




\section{DISCUSIÓN}

En el presente artículo de revisión sistemática, se organizó la información encontrada en las diversas publicaciones, coincidiendo con los autores Montenegro (2007), Gálvez y Milla (2018), Hernández y Ceniceros (2018), el desempeño docente es un conjunto de acciones realizadas por el docente, que comprende muchos aspectos, en una relación docente, estudiante y entorno. Las funciones relacionadas a la labor docente comprenden la planificación de clase, ejecución de la enseñanza, articulación con la comunidad y la formación continua.

Además, se ha encontrado sobre los artículos revisados, Perú tiene mayor cantidad de artículos sobre el desempeño docente. Con respecto a las publicaciones realizadas por las diferentes bases de datos, se encuentra mayor porcentaje en Scielo es la que presenta mayor porcentaje de publicaciones realizadas sobre el tema de estudio.

Asimismo, las investigaciones realizadas sobre el desempeño docente, corresponde en mayor porcentaje al nivel secundaria, debiéndose prestar atención también a los otros niveles educativos, puesto que en todos los niveles es importante el desempeño docente.

Finalmente, los hallazgos recogidos de las investigaciones realizadas destacan que el buen desempeño docente implica múltiples aspectos a ser considerados tales como, la planificación, motivación del docente, manejo de estrategias de enseñanza, la gestión del directivo, uso de recursos enfatizando en la tecnología y el monitoreo y acompañamiento como asesoría especializada que le permita reflexionar e identificar dificultades para emprender un proceso de cambio.

\section{Conclusiones}


La revisión de los diversos artículos, ensayos, autores referentes del desempeño docente, muestra que Perú, cuenta con mayor número de artículos publicados en relación al tema de estudio y en las bases de datos revisadas.

Se afirma que de acuerdo a la a la revisión sistemática, de las diversas investigaciones sobre el desempeño docente, es definido tomando en cuenta diversos aspectos que involucra en dicha labor, tales como, la preparación de la clase que implica conocer el contexto y características de los estudiantes, en la ejecución de la clase, tomar en cuenta el uso de recursos y estrategias de aprendizaje y la evaluación, sin dejar de lado la gestión directiva, enfatizando en el apoyo pedagógico a los maestros, aplicando el monitoreo y acompañamiento, mediante la asesoría especializada cuando se detecta dificultades en la práctica pedagógica y finalmente la formación continua del docente para la mejora de su desempeño.

\section{REFERENCIAS}

Achata, C., y Quispe, Y. (2018). El currículo nacional y el desempeño docente. Revista de investigaciones, $7(2), \quad 598-606$, https://doi.org/10.26788/epg.v7i2.321

Agreda, A., y Pérez, M. (2020). Relación entre acompañamiento pedagógico y practica reflexiva. Espacios en blanco. Serie Indagaciones , 30(2), 1-10. http://www.scielo.org.ar/scielo.php?script=sci_arttext\&pid=S151594852020000200001\&lng=es\&tlng=es.

Álvarez, G., (2020). Evaluación del desempeño docente en Instituciones Educativas Públicas. Eduser, 7(1), 32-40, DOI 10.18050/eduser.v7i1.2423 BID, (2020). Informe del BID, revela problemas en América Latina y el Caribe. https:/www.iadb.org/es/noticias/informe-del-bidrevela-problemas-de-evaluacion-docente-en-america-latina-y-el-caribe Comunicado Prensa 24 febrero 2020 
Casas, A. (2019). Liderazgo pedagógico, nuevas perspectivas para el desempeño docente. Investigación Valdizana, 13(1), 51-59. https://doi.org/10.33554/riv.13.1.171.

Chenet, M., Arévalo, J. y Palma, F. (2017). Identidad cultural y desempeño docente en las instituciones educativas. Opción, 33(84), 292-322. https://www.redalyc.org/articulo.oa? $\mathrm{id}=31054991011$

Cuenca, R. (2011). Hacia una propuesta del buen desempeño docente. Instituto de Estudios Peruanos.https://repositorio.iep.org.pe/bitstream/handle/IEP/725/cuenca_dsc ursosynociones.pdf;jsessionid=9FC2243151ABF05BC2F699127D4ECD67 ?sequence $=2$

De la Cruz, R. (2017). Liderazgo transformacional y desempeño docente en el Cepre de la universidad Nacional del Centro del Perú. Socialium, 2(2), 3447. DOI: https://doi.org/10.31876/sl.v1i1.2

Escribano, E. (2018). El desempeño docente como factor asociado a la calidad educativa en América latina. Revista Educación. 42(2), 717-739, https://doi.org/10.15517/revedu.v42i2.27033

Fuentes, A., López, J., y Pozo, S. (2019). Análisis de la competencia digital docente factor clave en el desempeño de pedagogías activas con realidad aumentada. Revista Iberoamericana sobre Calidad, Eficacia y cambio en Educación, 17 (2) https://doi.org/10.15366/reice2019.17.2.002

Galán, P., Hernández, R., y Espinoza, A. (2020). Enseñar con tecnologías digitales en la educación superior. Revista Perspectivas, (17), 1-12. https://revistas.uniminuto.edu/index.php/Pers/article/view/2129

Gálvez, E., y Milla., R. (2018). Evaluación del desempeño docente: Preparación para el aprendizaje de los estudiantes en el Marco del Buen 
Desempeño Docente. Propósitos y Representaciones, 6(2), 407-429, https://doi.org/10.20511/pyr2018.v6n2.236

Gallardo, M., y Pacheco, C. (2019). Evaluación docente y desempeño profesional: en juego la condición afectiva del profesorado. Revista de la Escuela de Ciencias de la Educación, 2(14), 81-89, http://www.scielo.org.ar/scielo.php?script=sci_arttext\&pid=S236233492019000200009\&lng=es\&tlng=es.

García, M., (2020). Relación entre la ejecución curricular y el desempeño docente. Investigación Valdizana, 14(2), 103-111, https://doi.org/10.33554/riv.14.2.698

Geraldo, L., Soria, J., Roselló, M., y Buendía, K. (2021). Modelo factorial en la motivación laboral y desempeño docente. Caso. Institución Educativa Emblemática Isabel La Católica. Apuntes Universitarios, 11(1), https://doi.org/10.17162/au.v11i1.561

Gómez, L., y Valdés, M. (2019). La evaluación del desempeño docente en la educación superior. Propósitos y Representaciones, 7(2), 479-515

Gonzáles, M., (2019) La educación en un mundo global. Revista Tecnológica Ciencia y Educación Edwards Deming, 3(1), 13-35.

Granados, J., Pilay, L., Ortiz, M., y Guadalupe, W. (2019). El docente universitario potenciando el aprendizaje ubicuo mediante el empleo de plataformas de gestión. Espirales, 3(24), 159-166. https://doi.org/10.31876/er.v3i24.649

Haddad, L., y Barbosa, A. (2020). Lições de quarentena : límites e posibilidades da atuação docente en época de isolamento social. Práxis Educativa, 15(15483), 1-24.

https://doi.org/10.5212/PraxEduc.v.15.15483.076 
Hernández, L., y Ceniceros, D. (2018). Autoeficacia docente y desempeño docente ¿una relación entre variables?, Innovación Educativa, 18(78), 171192 ,

López, E., García, L., y Martínez, J. (2019). La gestión directiva como potenciadora de la mejora del clima organizacional y la convivencia en las instituciones de educación media superior. Revista Iberoamericana para la investigación y el Desarrollo Educativo, 9(18), 792-812, https://doi.org/10.23913/ride.v9i18.471

Martínez, G., Esparza, A., y Gómez, R., (2021). El desempeño docente desde la perspectiva de la práctica profesional. Revista Iberoamericana de Investigación $\quad \mathrm{y} \quad$ Desarrollo Educativo, $11(21)$, https://doi.org/10.23913/ride.v11i21.703

Mendoza, L. (2020). Lo que la pandemia nos enseñó sobre la educación a distancia. Revista latinoamericana de estudios educativos, 1, 343-352, https://doi.org/10.48102/rlee.2020.50.ESPECIAL.119

Meza, L., Torres, J., y Mamani, B. (2021). Gestión educativa como factor determinante del desempeño de los docentes de educación básica regular durante la pandemia COVID-19, Puno-Perú. Apuntes Universitarios, 11(1), 23-35, https://doi.org/10.17162/au.v11i1.543

MINEDU (2012). Marco del Buen Desempeño Docente http://www.minedu.gob.pe/n/xtras/marco_buen_desempeno_docente.pdf Minedu (2019). Evaluación docente. https://evaluaciondocente.perueduca.pe/ascenso2021/

Montenegro, I. (2007). Evaluación del desempeño docente. Cooperativa Editorial Magisterio. Segunda edición. Bogotá Colombia. https://books.google.com.pe/books?id=8mH8ozUEIL4C\&printsec=frontcov er\&hl $=$ es\&source $=\mathrm{gbs} \_g e \_s u m m a r y \_r \& c a d=0 \# \mathrm{v}=$ onepage $\& \mathrm{q} \& \mathrm{f}=$ false 
More, R., y Morey, M. (2021). Gestión del cima institucional y desempeño del personal docente de la Institución Educativa No 7044 san martín de Porres, Chorrillos. Dilemas Contemporáneos: Educación política y valores, 1(3), https://doi.org/10.46377/dilemas.v8i.2588

Murillo, P. (2008 b). La autoevaluación institucional: Un camino importante para la mejora de los centros educativos.

Quispe, M. (2020).La gestión pedagógica en la mejora del desempeño docente. Investigación $\quad$ Valdizana, $14(19, \quad 7-14$, https://doi.org/10.33554/riv.14.1.601

Soria, L., Ortega, W., y Ortega, A. (2020). Desempeño pedagógico docente y aprendizaje de los estudiantes universitarios en la carrera de Educación. $\begin{array}{llll}\text { Praxis } \quad \& \quad \text { Saber, } & \text { 11(27), }\end{array}$ https://doi.org/10.19053/22160159.v11.n27.2020.10329

Trujillo, P., Ortiz, M., Carrasco, M., Herrera, G., y Martínez, C. (2020). Intercambios, 7(2), 66-78, http://dx.doi.org/10.2916/inter.7.2.8.

OREALC/UNESCO Santiago (2007). Educación de calidad para todos: un asunto de derechos humanos. Documento de discusión sobre políticas educativas en el marco de la II Reunión Intergubernamental del Proyecto Regional de Educación para América Latina y el Caribe (EPT/PRELAC). Santiago de Chile, OREALC/UNESCO. https://www.fundacionhenrydunant.org/images/stories/biblioteca/derecho-ala-

educacion/Educacion_de_calidad_para_todos_un_asunto_de_derechos $\% 20 \mathrm{~h}$ umanos.pdf

OREAL/UNESCO (2013). Antecedentes y Criterios para la elaboración de políticas docentes en América Latina y el Caribe. Ediciones UNESCO 7. CHILE 
Osorio, S. (2021). Hacia una transformación docente con mentalidad de crecimiento y resiliencia. Universciencia. 19(56), 43-50, http://revista.soyuo.mx/index.php/uc

Peniche, R., Ramón, C., Guzmán, C., y Mora, N. (2020.) Factores que afectan el desempeño docente en centros de alta y baja eficacia en México. Revista Iberoamericana sobre Calidad, Eficacia y Cambio en Educación, 18(2), 7795 https://doi.org/10.15366/reice2020.18.2.004

Picón, G., Gonzales, G., y Paredes, j. (2020.) Desempeño y formación docente en competencias digitales en clases no presenciales durante la pandemia COVID-19. Investigación Educativa, https://doi.org/10.1590/SciELOPreprints.778

Puertas, P., Ubago, J., Moreno., Padial, R., y Martínez, A., (2018). La inteligencia emocional en la formación y desempeño docente: Una revisión sistemática. Asociación Española de Orientación y Psicopedagógica, 29(2), https://doi.org/10.5944/reop.vol.29.num.2.2018.23157

Rodríguez, k., y Lechuga, J. (2019). Desempeño laboral de los docentes de la Institución Universitaria ITSA. AEASCUELA .79-101 https://www.redalyc.org/jatsRepo/206/20663246005/20663246005.pdf

Soria, Ll., Ortega W., Ortega A, (2021). Desempeño pedagógico docente y aprendizaje de los estudiantes universitarios en la carrera de Educación. Praxis \& Saber https://doi.org/10.19053/22160159.v11.n27.2020.10329 Urrutia, F. (2020). Desempeño docente en la formación cívica y ética: estudio empírico y recomendaciones de política educativa para la escuela secundaria básica en México. Archivos analíticos de políticas educativas, 28(188), 1-29, https://doi.org/10.14507/epaa.28.5255 
Valcazar. G (2020). El desempeño docente y la práctica inclusiva en el nivel superior. Desde el Sur, 12(2), 437-452, https://doi.org/10.21142/DES-12022020-0025 